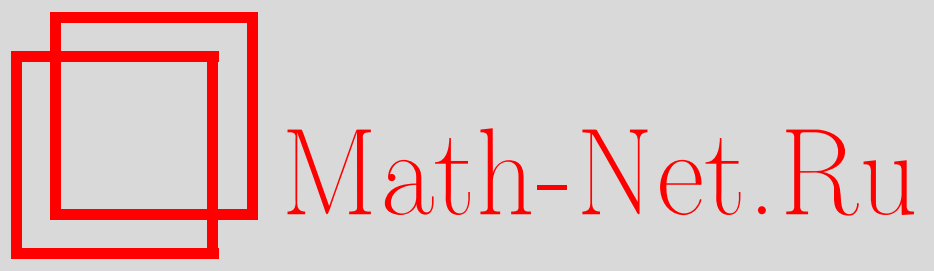

Ж. Х. Петров, Д. И. Толев, Об одном уравнении включающем дробные степени с одной простой и одной почти простой переменными, Совр. пробл. матем., 2017, выпуск 24, 46-65

DOI: https://doi.org/10.4213/spm68

Использование Общероссийского математического портала Math-Net.Ru подразумевает, что вы прочитали и согласны с пользовательским соглашением http://www.mathnet.ru/rus/agreement

Параметры загрузки:

IP : 107.22 .136 .117

26 апреля 2023 г., 14:04:52

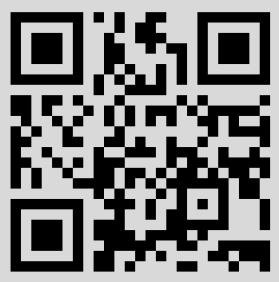




\title{
Об одном уравнении, включающем дробные степени \\ с одной простой и одной почти простой переменными
}

\author{
Ж. Х. Петров, Д. И. Толев \\ Sofia University St. Kliment Ohridski, Bulgaria \\ zhpetrov@fmi.uni-sofia.bg, dtolev@fmi.uni-sofia.bg
}

Посвящаяется памяти профессора А. А. Карачубъ

\section{1. Введение и формулировка результата}

В 1953 г. Пятецкий-Шапиро [1] доказал, что если константа $c \in \mathbb{R}$ удовлетворяет неравенствам $1<c<12 / 11$, то существует бесконечно много простых вида

$$
\left[n^{c}\right], \quad \text { где } n \in \mathbb{N},
$$

и где, как обычно, $[t]$ означает целую часть $t$. После верхняя граница для $c$ была много раз улучшена, и самый сильный результат принадлежит Рива и Ву [2]. Они доказали, что то же самое утверждение справедливо при условии, что $1<c<243 / 205$.

При любом натуральном $r$ обозначим через $\mathcal{P}_{r}$ множество почти простых чисел порядка $r$, т.е. множество натуральных чисел, состоящих не более чем из $r$ простых сомножителей с учетом кратности.

Есть много работ, посвященных изучению проблем, связанных с простыми числами Пятецкого-Шапиро и с почти простыми числами. В 2011 г. Кай и Ван [3], улучшая более ранний результат Пеневой [4], показали, что если $1<c<30 / 29$, то существует бесконечно много простых чисел $p$ вида $\left[n^{c}\right]$ таких, что $p+2 \in \mathcal{P}_{5}$. Позднее, в 2014 г., Бейкер, Банкс, Го и Йегер [5] показали, что если $1<c<77 / 76$, то последовательность

$$
\left\{\left[n^{c}\right]\right\}_{n \in \mathcal{P}_{8}}
$$

содержит бесконечно много простых чисел.

Рассмотрим уравнение

$$
\left[m_{1}^{c}\right]+\left[m_{2}^{c}\right]=N .
$$

В 1973 г. Дезуйе [6] доказал, что если $1<c<4 / 3$, то для любого достаточно большого целого числа $N$ уравнение $(2)$ с целыми $m_{1}$ и $m_{2}$ имеет решение. Этот результат был улучшен Гриценко [7], а затем Конягиным [8]. В частности, последний автор показал, что (2) имеет решение в целых числах $m_{1}, m_{2}$ при $1<c<3 / 2$ и достаточно большом $N$.

Кумчев [9] доказал, что если $1<c<16 / 15$, то всякое достаточно большое целое число $N$ может быть представлено в виде (2), где $m_{1}$ является простым, а $m_{2}$ целым числом. С другой стороны, знаменитая теорема Чена [10] утверждает, что любое достаточно большое четное целое может быть представлено в виде суммы простого и почти простого из $\mathcal{P}_{2}$. Имея в виду этот глубокий результат, можно предположить, что существует такая постоянная $c_{0}>1$, что при $1<c<c_{0}$ уравнение (2) имеет решение с простым $m_{1}$ и $m_{2} \in \mathcal{P}_{2}$ при условии, что $N$ достаточно велико.

В настоящей работе мы устанавливаем результат такого типа и доказываем следующее утверждение.

Работа выполнена при поддержке Софийского университета (грант SU 113/2016).

(C) Ж. Х. Петров, Д. И. Толев, 2017 
Теорема. Предположим, что $1<c<29 / 28$. Тогда каждое достаточно большое иелое $N$ может быть представлено как

$$
\left[p^{c}\right]+\left[m^{c}\right]=N,
$$

где р есть простое и т есть почти простое число с не более чем $[52 /(29-28 c)]+1$ простыми сомножителями.

Заметим, что целое число $[52 /(29-28 c)]+1$ равняется 53 , если с близко к 1 , и становится большим, если с близко к 29/28.

Наш первый шаг в доказательстве состоит в применении линейного решета. После этого, мы могли бы попытаться установить сравнительно сильную оценку для экспоненциальной суммы, определенной в (31), что является довольно сложной задачей, так как функция в показателе степени зависит от $\left[p^{c}\right]$. Вместо этого мы представим эту сумму в виде линейной комбинации подобных сумм (см. (60)) с гладкой функцией аргумента $p$ в показателе. Затем мы используем стандартные методы для оценки этих сумм. Мы хотели бы отметить, что суммы вида (60) изучались также Кумчевым [9]. Однако мы не можем использовать его работу, потому что нам требуются более сильные оценки для них.

\section{2. Обозначения}

Введем следующие обозначения: $\{t\}$ есть дробная часть числа $t$, функция $\rho(t)$ определяется как $\rho(t)=1 / 2-\{t\}$ и $e(t)=e^{2 \pi i t}$. Символ $p$ будет зарезервирован для простых чисел. Через $\varepsilon$ мы обозначим сколь угодно малое положительное число, не обязательно одно и то же в разных формулах. Мы пользуемся обозначением Виноградова $A \ll B$, которое эквивалентно $A=$ $O(B)$. Если у нас одновременно $A \ll B$ и $B \ll A$, то будем писать $A \asymp B$. Подразумевается, что константы являются абсолютными или зависят от $c$ или $\varepsilon$.

Как обычно, $\sum_{n \leqslant x}$ значит $\sum_{1 \leqslant n \leqslant x}$ и $\mu(n), \Lambda(n)$ и $\tau(n)$ являются функцией Мебиуса, функцией фон Мангольдта и числом положительных делителей $n$ соответственно.

\section{3. Доказательство теоремы}

3.1. Начало доказательства. Пусть $N$ - достаточно большое целое, и пусть

$$
1<c<\frac{29}{28}, \quad \gamma=\frac{1}{c}, \quad P=10^{-9} N^{\gamma} .
$$

Предположим, что $\alpha>0$ есть константа, которая будет определена позднее, и пусть

$$
z=N^{\alpha}, \quad B_{z}=\prod_{p<z} p .
$$

Рассмотрим сумму

$$
\Gamma=\sum_{\substack{P<p \leqslant 2 P, m \in \mathbb{N} \\\left[p^{c}\right]+\left[m^{c}\right]=N \\\left(m, B_{z}\right)=1}} \log p .
$$

Если $\Gamma>0$, то найдутся простое число $p$ и натуральное число $m$, удовлетворяющие введенным условиям в области суммирования Г. Из условия $\left(m, B_{z}\right)=1$ следует, что любой простой делитель $m$ не меньше чем $z$. Предположим, что $m$ состоит из $l$ простых сомножителей, учитываемых с кратностью. Тогда имеем

$$
N^{\gamma} \geqslant m \geqslant z^{l}=N^{\alpha l},
$$

и отсюда $l \leqslant \gamma / \alpha$. Из этого следует, что если $\Gamma>0$, то $(3)$ имеет решение с простым $p$ и почти простым $m$, состоящим не более чем из $[\gamma / \alpha]$ простых сомножителей. 
Обозначим

$$
D=N^{\delta}
$$

где $\delta>0$ - это константа, которая будет определена позднее. Обозначим через $\lambda(d)$ нижний россеровский вес уровня $D$ (см. [11; гл. 4]). Тогда имеем

$$
\sum_{d \mid k} \mu(d) \geqslant \sum_{d \mid k} \lambda(d) \quad \text { для любого } \quad k \in \mathbb{N} \text {. }
$$

Кроме того, мы знаем, что

$$
\begin{array}{rr}
|\lambda(d)| \leqslant 1 & \text { для всех } \quad d, \\
\lambda(d)=0 \quad \text { если } \quad d>D \quad \text { или } \quad \mu(d)=0 .
\end{array}
$$

Наконец, имеем

$$
\sum_{d \mid B_{z}} \frac{\lambda(d)}{d} \geqslant \prod_{p<z}\left(1-\frac{1}{p}\right)\left(f\left(s_{0}\right)+O\left((\log D)^{-1 / 3}\right)\right)
$$

где

$$
s_{0}=\frac{\log D}{\log z}=\frac{\delta}{\alpha}
$$

и $f(s)$ является нижней функцией линейного решета, для которой известно, что

$$
f(s)= \begin{cases}0 & \text { при } 0<s<2, \\ 2 e^{G} s^{-1} \log (s-1) & \text { при } 2<s<3\end{cases}
$$

здесь $G$ - константа Эйлера.

Из (6) и (8) находим

$$
\Gamma=\sum_{\substack{P<p \leqslant 2 P, m \in \mathbb{N} \\\left[p^{c}\right]+\left[m^{c}\right]=N}}(\log p) \sum_{d \mid\left(m, B_{z}\right)} \mu(d) \geqslant \sum_{\substack{P<p \leqslant 2 P, m \in \mathbb{N} \\\left[p^{c}\right]+\left[m^{c}\right]=N}}(\log p) \sum_{d \mid\left(m, B_{z}\right)} \lambda(d) .
$$

Изменим порядок суммирования и получим

$$
\Gamma \geqslant \sum_{d \mid B_{z}} \lambda(d) G_{d}, \quad \text { где } \quad G_{d}=\sum_{\substack{P<p \leqslant 2 P, m \in \mathbb{N} \\\left[p^{c}\right]+\left[m^{c}\right]=N \\ m \equiv 0(\bmod d)}} \log p .
$$

Теперь запишем сумму $G_{d}$ в виде

$$
G_{d}=\sum_{P<p \leqslant 2 P}(\log p) G_{d, p}^{\prime}, \quad \text { где } \quad G_{d, p}^{\prime}=\sum_{\substack{m \in \mathbb{N} \\ m=0(\bmod d) \\\left[p^{c}\right]+\left[m^{c}\right]=N}} 1 .
$$

Мы пользуемся очевидным тождеством

$$
\sum_{a \leqslant m<b} 1=[-a]-[-b]=b-a-\rho(-b)+\rho(-a),
$$

чтобы установить, что

$$
\begin{aligned}
G_{d, p}^{\prime}= & \sum_{\substack{m \in \mathbb{N} \\
m \equiv 0(\bmod d) \\
N-\left[p^{c}\right] \leqslant m^{c}<N+1-\left[p^{c}\right]}} 1=\sum_{(1 / d)\left(N-\left[p^{c}\right]\right)^{\gamma} \leqslant m<(1 / d)\left(N+1-\left[p^{c}\right]\right)^{\gamma}} 1 \\
= & \frac{\left(N+1-\left[p^{c}\right]\right)^{\gamma}-\left(N-\left[p^{c}\right]\right)^{\gamma}}{d}+\rho\left(-\frac{1}{d}\left(N-\left[p^{c}\right]\right)^{\gamma}\right)-\rho\left(-\frac{1}{d}\left(N+1-\left[p^{c}\right]\right)^{\gamma}\right) .
\end{aligned}
$$


Объединим вышеприведенное с (14), чтобы получить

$$
G_{d}=\frac{1}{d} A(N)+\sum_{P<p \leqslant 2 P}(\log p)\left(\rho\left(-\frac{1}{d}\left(N-\left[p^{c}\right]\right)^{\gamma}\right)-\rho\left(-\frac{1}{d}\left(N+1-\left[p^{c}\right]\right)^{\gamma}\right)\right),
$$

где

$$
A(N)=\sum_{P<p \leqslant 2 P}(\log p)\left(\left(N-\left[p^{c}\right]+1\right)^{\gamma}-\left(N-\left[p^{c}\right]\right)^{\gamma}\right)
$$

Из

$$
\left(N-\left[p^{c}\right]+1\right)^{\gamma}=\left(N-\left[p^{c}\right]\right)^{\gamma}+\gamma\left(N-\left[p^{c}\right]\right)^{\gamma-1}+O\left(N^{\gamma-2}\right)
$$

получаем, что

$$
A(N)=\gamma \sum_{P<p \leqslant 2 P}(\log p)\left(\left(N-\left[p^{c}\right]\right)^{\gamma-1}+O\left(N^{\gamma-2}\right)\right),
$$

и по теореме о распределении простых чисел Чебышёва и определения $P$ из (4) получаем

$$
A(N) \asymp N^{\gamma-1} \sum_{P<p \leqslant 2 P} \log p \asymp N^{2 \gamma-1} .
$$

Из (13) и (15) имеем

$$
\Gamma \geqslant \Gamma_{0}+\Sigma_{0}-\Sigma_{1}
$$

где

$$
\begin{gathered}
\Gamma_{0}=A(N) \sum_{d \mid B_{z}} \frac{\lambda(d)}{d}, \\
\Sigma_{j}=\sum_{d \mid B_{z}} \lambda(d) \sum_{P<p \leqslant 2 P}(\log p) \rho\left(-\frac{1}{d}\left(N+j-\left[p^{c}\right]\right)^{\gamma}\right), \quad j=0,1 .
\end{gathered}
$$

Рассмотрим $\Gamma_{0}$. Пользуясь (5) и формулой Мертенса, находим

$$
\prod_{p<z}\left(1-\frac{1}{p}\right) \asymp \frac{1}{\log z} \asymp \frac{1}{\log N} .
$$

Предположим, что

$$
2<\frac{\delta}{\alpha}<3
$$

Тогда, имея в виду (11) и (12), находим, что $f\left(s_{0}\right)>\kappa$ при некоторой константе $\kappa>0$, зависящей только от $\delta$ и $\alpha$. (Однако $\delta$ и $\alpha$ зависят только от $c$, так что $\kappa$ также зависит только от с.) Следовательно, пользуясь (10) и (20) получаем

$$
\sum_{d \mid B_{z}} \frac{\lambda(d)}{d} \gg \frac{1}{\log N} .
$$

Таким образом, из (16) и (18) получим

$$
\Gamma_{0} \gg \frac{N^{2 \gamma-1}}{\log N} .
$$

Мы стремимся установить следующую оценку для сумм $\Sigma_{j}$, определенных в (19):

$$
\Sigma_{j} \ll \frac{N^{2 \gamma-1}}{(\log N)^{2}}, \quad j=0,1 .
$$


Вместе с (17) и (22) это будет значить, что

$$
\Gamma \gg \frac{N^{2 \gamma-1}}{\log N},
$$

откуда $\Gamma>0$ для достаточно большого $N$. Тогда, как мы уже объяснили, уравнение (3) будет иметь решение с простым $p$ и почти простым $m$ не более чем с $[\gamma / \alpha]$ простыми сомножителями.

Оставшаяся часть статьи будет посвящена доказательству оценок (23) в предположениях

$$
\frac{28}{29}<\gamma<1, \quad \delta<\frac{29 \gamma-28}{26} .
$$

Затем останется выбрать

$$
\alpha=\frac{29 \gamma-28}{52}-\varepsilon_{0}
$$

для некоторого малого $\varepsilon_{0}>0$ и взять

$$
\delta \in\left(2 \alpha, \frac{29 \gamma-28}{26}\right) .
$$

В этом случае, когда $\varepsilon_{0}$ достаточно мало, условие (21) выполняется. Выбирая с на основе (4), легко видеть, что

$$
\left[\frac{\gamma}{\alpha}\right] \leqslant\left[\frac{52}{29-28 c}\right]+1
$$

что доказывает теорему.

3.2. Оценка сумм $\boldsymbol{\Sigma}_{\mathbf{1}}$ и $\boldsymbol{\Sigma}_{\mathbf{2}}$ (начало). Рассмотрим сумму $\Sigma_{j}$, определенную в (19). Применим теорему Ваалера [12], в которой говорится, что для каждого $H \geqslant 2$ найдутся такие числа $c_{h}, 0<|h| \leqslant H$, и $d_{h},|h| \leqslant H$, что

$$
\rho(t)=\sum_{0<|h| \leqslant H} c_{h} e(h t)+\Delta_{H}(t)
$$

где

$$
\left|\Delta_{H}(t)\right| \leqslant \sum_{|h| \leqslant H} d_{h} e(h t)
$$

И

$$
\left|c_{h}\right| \ll \frac{1}{|h|}, \quad\left|d_{h}\right| \ll \frac{1}{H} .
$$

Из (19) и (25) следует, что

$$
\Sigma_{j}=\Sigma_{j}^{\prime}+\Sigma_{j}^{\prime \prime},
$$

где

$$
\begin{aligned}
\Sigma_{j}^{\prime} & =\sum_{d \leqslant D} \lambda(d) \sum_{P<p \leqslant 2 P}(\log p) \sum_{0<|h| \leqslant H} c_{h} e\left(-\frac{h}{d}\left(N+j-\left[p^{c}\right]\right)^{\gamma}\right), \\
\Sigma_{j}^{\prime \prime} & =\sum_{d \leqslant D} \lambda(d) \sum_{P<p \leqslant 2 P}(\log p) \Delta_{H}\left(-\frac{\left(N+j-\left[p^{c}\right]\right)^{\gamma}}{d}\right) .
\end{aligned}
$$

Пусть

$$
W(v)=\sum_{P<p \leqslant 2 P}(\log p) e\left(v\left(N+j-\left[p^{c}\right]\right)^{\gamma}\right) .
$$


Начнем с суммы $\Sigma_{j}^{\prime}$. Изменение порядка суммирования вместе с $(9),(27)$ и (31) влечет

$$
\Sigma_{j}^{\prime}=\sum_{d \leqslant D} \lambda(d) \sum_{0<|h| \leqslant H} c_{h} W\left(-\frac{h}{d}\right) \ll \sum_{d \leqslant D} \sum_{1 \leqslant h \leqslant H} \frac{1}{h}\left|W\left(\frac{h}{d}\right)\right| .
$$

Для суммы $\Sigma_{j}^{\prime \prime}$ воспользуемся (9), (26), (27) и (31), чтобы получить

$$
\begin{aligned}
\Sigma_{j}^{\prime \prime} & \ll \sum_{d \leqslant D} \sum_{P<p \leqslant 2 P}(\log p) \sum_{|h| \leqslant H} d_{h} e\left(-\frac{h}{d}\left(N+j-\left[p^{c}\right]\right)^{\gamma}\right) \\
& =\sum_{d \leqslant D} \sum_{|h| \leqslant H} d_{h} W\left(-\frac{h}{d}\right) \ll \sum_{d \leqslant D} \sum_{|h| \leqslant H} \frac{1}{H}\left|W\left(\frac{h}{d}\right)\right| .
\end{aligned}
$$

Из (4), (31) и теоремы Чебышёва о распределении простых чисел находим, что $W(0) \asymp N^{\gamma}$ и отсюда

$$
\Sigma_{j}^{\prime \prime} \ll \sum_{d \leqslant D} \frac{N^{\gamma}}{H}+\sum_{d \leqslant D} \sum_{1 \leqslant h \leqslant H} \frac{1}{H}\left|W\left(\frac{h}{d}\right)\right| .
$$

Положим

$$
H=d N^{1-\gamma}(\log N)^{3} .
$$

Теперь, пользуясь (7), (28) и (32)-(34), получим

$$
\Sigma_{j} \ll \frac{N^{2 \gamma-1}}{(\log N)^{2}}+\sum_{d \leqslant D} \sum_{h \leqslant H} \frac{1}{h}\left|W\left(\frac{h}{d}\right)\right|, \quad j=0,1 .
$$

3.3. Изучение суммы $\boldsymbol{W}(\boldsymbol{v})$. Как мы уже упоминали ранее, трудно оценить экспоненциальную сумму $W(v)$, определяемую (31), непосредственно. Вместо этого мы можем записать ее в виде линейной комбинации аналогичных сумм, с которыми легче иметь дело.

Пусть $Z \geqslant 2$ - целое, которое мы определим позднее. Применим известную лемму "о стаканчиках" Виноградова (см. [13; гл. 1, лемма А]) с параметрами

$$
\alpha=-\frac{1}{4 Z}, \quad \beta=\frac{1}{4 Z}, \quad \Delta=\frac{1}{2 Z}, \quad r=[\log N]
$$

и построим функцию $g(t)$, периодическую с периодом 1 и обладающую следующими свойствами:

$$
\begin{gathered}
g(0)=1, \\
0<g(t)<1 \quad \text { при } \quad 0<|t|<\frac{1}{2 Z}, \\
g(t)=0 \quad \text { при } \quad \frac{1}{2 Z} \leqslant|t| \leqslant \frac{1}{2} .
\end{gathered}
$$

Кроме того, ряд Фурье $g(t)$ задается как

$$
g(t)=\frac{1}{2 Z}+\sum_{\substack{n \in \mathbb{Z} \\ n \neq 0}} \beta_{n} e(n t) \quad \text { при } \quad\left|\beta_{n}\right| \leqslant \min \left(\frac{1}{2 Z}, \frac{1}{|n|}\left(\frac{2 Z[\log N]}{\pi|n|}\right)^{[\log N]}\right) .
$$

Из вышеприведенной оценки $\left|\beta_{n}\right|$ легко получается

$$
\sum_{|n|>Z(\log N)^{4}}\left|\beta_{n}\right| \ll N^{-\log \log N}
$$


с некоторой абсолютной константой в знаке «. Отсюда имеем

$$
g(t)=\sum_{|n| \leqslant Z(\log N)^{4}} \beta_{n} e(n t)+O\left(N^{-\log \log N}\right),
$$

где подразумеваемая константа является абсолютной и

$$
\left|\beta_{n}\right| \leqslant \frac{1}{2 Z}
$$

Наконец, легко видеть, что функция $g(t)$, построенная в доказательстве леммы А из [13; гл. 1], четная и также удовлетворяет

$$
g(t)+g\left(t-\frac{1}{2 Z}\right)=1 \quad \text { при } \quad 0 \leqslant t \leqslant \frac{1}{2 Z} .
$$

Пусть

$$
g_{z}(t)=g\left(t-\frac{z}{2 Z}\right) \quad \text { при } \quad z=0,1,2, \ldots, 2 Z-1 .
$$

Очевидно, что каждая функция $g_{z}(t)$ является периодической с периодом 1. Из (36) находим, что

$$
\begin{aligned}
& 0<g_{z}(t) \leqslant 1, \quad \text { если }\left|t-\frac{z}{2 Z}\right|<\frac{1}{2 Z}, \\
& g_{z}(t)=0, \quad \text { если } \quad \frac{1}{2 Z} \leqslant\left|t-\frac{z}{2 Z}\right| \leqslant \frac{1}{2} .
\end{aligned}
$$

Из (40) следует, что если $\beta_{n}^{(z)}$ есть $n$-й коэффициент Фурье функции $g_{z}(t)$, то

$$
\beta_{n}^{(z)}=\beta_{n} e\left(-\frac{z n}{2 M}\right)
$$

и отсюда $\left|\beta_{n}^{(z)}\right|=\left|\beta_{n}\right|$. Из этого наблюдения и из (37), а также вышеприведенной оценки для $\left|\beta_{n}\right|$ находим, что для $z=0,1, \ldots, 2 Z-1$ справедливо соотношение

$$
g_{z}(t)=\sum_{|n| \leqslant Z(\log N)^{4}} \beta_{n}^{(z)} e(n t)+O\left(N^{-\log \log N}\right),
$$

где константа в символе $O$ является абсолютной и

$$
\left|\beta_{n}^{(z)}\right| \leqslant \frac{1}{2 Z}
$$

Наконец, из (39), (40) и (42) находим

$$
\sum_{z=0}^{2 Z-1} g_{z}(t)=1 \quad \text { для всех } t \in \mathbb{R} .
$$

(Оставляем эту легкую проверку читателю.)

Теперь рассмотрим сумму $W(v)$, определенную в (31). Из (45) следует, что

$$
W(v)=\sum_{P<p \leqslant 2 P}(\log p) e\left(v\left(N+j-\left[p^{c}\right]\right)^{\gamma}\right) \sum_{z=0}^{2 Z-1} g_{z}\left(p^{c}\right)=\sum_{z=0}^{2 Z-1} W_{z}(v),
$$


где

$$
W_{z}(v)=\sum_{P<p \leqslant 2 P}(\log p) g_{z}\left(p^{c}\right) e\left(v\left(N+j-\left[p^{c}\right]\right)^{\gamma}\right) .
$$

Ясно, что

$$
W_{0}(v) \ll \sum_{P<p \leqslant 2 P}(\log p) g\left(p^{c}\right) \ll \log N \sum_{P<k \leqslant 2 P} g\left(k^{c}\right) .
$$

Применим (4), (37) и (38), чтобы получить

$$
\begin{aligned}
W_{0}(v) & \ll(\log N) \frac{P}{Z}+(\log N)\left|\sum_{P<k \leqslant 2 P} \sum_{0<|n| \leqslant Z(\log N)^{4}} \beta_{n} e\left(n k^{c}\right)\right|+1 \\
& \ll(\log N) \frac{N^{\gamma}}{Z}+\frac{\log N}{Z} \sum_{n \leqslant Z(\log N)^{4}}\left|\mathcal{H}_{n}\right|+1,
\end{aligned}
$$

где

$$
\mathcal{H}_{n}=\sum_{P<k \leqslant 2 P} e\left(n k^{c}\right)
$$

Если $\theta(x)=n x^{c}$, то $\theta^{\prime \prime}(x)=c(c-1) n x^{c-2} \asymp n P^{c-2}$ равномерно по $x \in[P, 2 P]$. Следовательно, можно применить теорему Ван дер Корпута (см. [13; гл. 1, теорема 5]), чтобы получить

$$
\mathcal{H}_{n} \ll P\left(n P^{c-2}\right)^{1 / 2}+\left(n P^{c-2}\right)^{-1 / 2} \ll P^{c / 2} n^{1 / 2} .
$$

В дальнейшем мы предполагаем, что

$$
Z \ll N^{(2 \gamma-1) / 3}(\log N)^{-4} .
$$

Тогда, пользуясь (4) и (48)-(50), получаем

$$
W_{0}(v) \ll(\log N)\left(\frac{N^{\gamma}}{Z}+N^{1 / 2} Z^{1 / 2} \log ^{6} N\right) \ll(\log N) \frac{N^{\gamma}}{Z} .
$$

Теперь мы ограничимся рассмотрением сумм $W_{z}(v)$ при $1 \leqslant z \leqslant 2 Z-1$. Из (42) видим, что $g_{z}\left(p^{c}\right)$ может быть отлично от нуля, только если $\left\{p^{c}\right\} \in[(z-1) /(2 Z),(z+1) /(2 Z)]$. Следовательно, единственные слагаемые в сумме (47) это те, для которых

$$
\left\{p^{c}\right\}=\frac{z}{2 Z}+O\left(\frac{1}{Z}\right) \text {. }
$$

В этом случае имеем

$$
v\left(N+j-\left[p^{c}\right]\right)^{\gamma}=v\left(N+j-p^{c}+\frac{z}{2 Z}\right)^{\gamma}+O\left(\frac{v N^{\gamma-1}}{Z}\right)
$$

и, соответственно,

$$
e\left(v\left(N+j-\left[p^{c}\right]\right)^{\gamma}\right)=e\left(v\left(N+j-p^{c}+\frac{z}{2 Z}\right)^{\gamma}\right)+O\left(\frac{v N^{\gamma-1}}{Z}\right) .
$$

Тогда, пользуясь (47), находим

$$
W_{z}(v)=V_{z}(v)+O\left(\frac{v N^{\gamma-1}}{Z} \sum_{P<p \leqslant 2 P}(\log p) g_{z}\left(p^{c}\right)\right),
$$


где

$$
V_{z}(v)=\sum_{P<p \leqslant 2 P}(\log p) g_{z}\left(p^{c}\right) e\left(v\left(N+j-p^{c}+\frac{z}{2 Z}\right)^{\gamma}\right) .
$$

Следовательно, из (46), (51) и (52) получаем

$$
W(v)=\sum_{z=1}^{2 Z-1} W_{z}(v)+W_{0}(v)=\sum_{z=1}^{2 Z-1} V_{z}(v)+O(\Xi)+O\left((\log N) \frac{N^{\gamma}}{Z}\right),
$$

где

$$
\Xi=\frac{v N^{\gamma-1}}{Z} \sum_{P<p \leqslant 2 P}(\log p) \sum_{z=1}^{2 Z-1} g_{z}\left(p^{c}\right) .
$$

Теперь пользуясь (4), (45) и теоремой Чебышёва о распределении простых чисел, находим, что

$$
\Xi \ll \frac{v N^{2 \gamma-1}}{Z}
$$

и, следовательно,

$$
W(v)=\sum_{z=1}^{2 Z-1} V_{z}(v)+O\left(\frac{v N^{2 \gamma-1}}{Z}\right)+O\left((\log N) \frac{N^{\gamma}}{Z}\right) .
$$

С этого момента и далее мы предполагаем, что

$$
v=\frac{h}{d}, \quad \text { где } \quad 1 \leqslant d \leqslant D, \quad 1 \leqslant h \leqslant H .
$$

Тогда из (34) видим, что $v N^{2 \gamma-1} \ll N^{\gamma}(\log N)^{3}$; отсюда формула (54) может быть записана как

$$
W(v)=\sum_{z=1}^{2 Z-1} V_{z}(v)+O\left((\log N)^{3} \frac{N^{\gamma}}{Z}\right) .
$$

Из (34), (35) и (56) находим

$$
\Sigma_{j} \ll \frac{N^{2 \gamma-1}}{(\log N)^{2}}+\sum_{d \leqslant D} \sum_{h \leqslant H} \frac{1}{h} \sum_{z=1}^{2 Z-1}\left|V_{z}(v)\right|+\sum_{d \leqslant D} \sum_{h \leqslant H} \frac{1}{h} \frac{N^{\gamma}}{Z}(\log N)^{3} .
$$

Выберем такое $Z$, что

$$
Z \asymp d N^{1-\gamma}(\log N)^{7}
$$

Из (7) и (24) следует, что условие (50) выполняется. Следовательно, из (57) и (58) находим

$$
\Sigma_{j} \ll \frac{N^{2 \gamma-1}}{(\log N)^{2}}+\sum_{d \leqslant D} \sum_{h \leqslant H} \frac{1}{h} \sum_{z=1}^{2 Z-1}\left|V_{z}(v)\right| .
$$

Рассмотрим теперь сумму $V_{z}(v)$, определенную в (53), где $v$ удовлетворяет (55). Из (43) находим, что для достаточно большого $N$ справедливо соотношение

$$
\begin{aligned}
V_{z}(v) & =\sum_{P<p \leqslant 2 P}(\log p)\left(\sum_{|r| \leqslant Z(\log N)^{4}} \beta_{r}^{(z)} e\left(r p^{c}\right)\right) e\left(v\left(N+j-p^{c}+\frac{z}{2 Z}\right)^{\gamma}\right)+O\left(N^{-10}\right) \\
& =\sum_{|r| \leqslant Z(\log N)^{4}} \beta_{r}^{(z)} U\left(N+j+\frac{z}{2 Z}, r, v\right)+O\left(N^{-10}\right),
\end{aligned}
$$


где

$$
U=U(T, r, v)=\sum_{P<p \leqslant 2 P}(\log p) e\left(r p^{c}+v\left(T-p^{c}\right)^{\gamma}\right) .
$$

Мы хотели бы отметить, что если $0 \leqslant j \leqslant 1$ и $1 \leqslant z \leqslant 2 Z-1$, то

$$
N+j+\frac{z}{2 Z} \in[N, N+2] \text {. }
$$

Кроме того, если мы также примем во внимание (44) и (58), мы получим

$$
V_{z}(v) \ll N^{-10}+\frac{1}{Z} \sum_{|r| \leqslant R} \sup _{T \in[N, N+2]}|U(T, r, v)|,
$$

где

$$
R=d N^{1-\gamma}(\log N)^{12}
$$

Если подставить вышеприведенное выражение для $V_{z}(v)$ в формулу $(59)$, то будем иметь

$$
\left|\Sigma_{1}\right|+\left|\Sigma_{2}\right| \ll \frac{N^{2 \gamma-1}}{(\log N)^{2}}+\sum_{d \leqslant D} \sum_{h \leqslant H} \frac{1}{h} \sum_{|r| \leqslant R} \sup _{T \in[N, N+2]}|U(T, r, v)| .
$$

3.4. Применение тождества Вона. Введем обозначения

$$
\begin{gathered}
\phi(t)=r t^{c}+v\left(T-t^{c}\right)^{\gamma} \\
f(m, l)=\phi(m l)=r(m l)^{c}+v\left(T-(m l)^{c}\right)^{\gamma} .
\end{gathered}
$$

Для суммы $U$, определенной в (60), имеем

$$
U=\sum_{P<n \leqslant 2 P} \Lambda(n) e(\phi(n))+O\left(P^{1 / 2}\right) .
$$

(Действительно, сумма в правой части приведенной выше формулы содержит все члены суммы $U$, а также члены, для которых $n=p^{\nu}, \nu \geqslant 2$, но можно легко убедиться, что их вклад $\ll P^{1 / 2}$.) Применяя теперь тождество Вона (см. [14]), находим, что

$$
U=U_{1}-U_{2}-U_{3}-U_{4}+O\left(P^{1 / 2}\right)
$$

где

$$
\begin{aligned}
U_{1} & =\sum_{m \leqslant P^{1 / 3}} \mu(m) \sum_{P / m<l \leqslant 2 P / m}(\log l) e(f(m, l)), \\
U_{2} & =\sum_{m \leqslant P^{1 / 3}} c(m) \sum_{P / m<l \leqslant 2 P / m} e(f(m, l)), \\
U_{3} & =\sum_{\substack{P^{1 / 3}<m \leqslant P^{2 / 3} \\
P / m<l \leqslant 2 P / m}} c(m) \sum_{P(m, l)),} a(m) \Lambda(l) e(f(m, l)) \\
U_{4} & =\sum_{\substack{P<m l \leqslant 2 P \\
m>P^{1 / 3}, l>P^{1 / 3}}} \sum_{\substack{ \\
m}} a(f(m)
\end{aligned}
$$

и

$$
|c(m)| \leqslant \log m, \quad|a(m)| \leqslant \tau(m) .
$$

Следовательно из (7), (24), (34), (61), (62) и (65) имеем

$$
\left|\Sigma_{1}\right|+\left|\Sigma_{2}\right| \ll \frac{N^{2 \gamma-1}}{(\log N)^{2}}+\sum_{i=1}^{4} \Omega_{i}
$$


где

$$
\Omega_{i}=\sum_{d \leqslant D} \sum_{h \leqslant H} \frac{1}{h} \sum_{|r| \leqslant R} \sup _{T \in[N, N+2]}\left|U_{i}\right| .
$$

Из (71) и (72), чтобы доказать, что удовлетворяется (23), достаточно показать, что

$$
\Omega_{i} \ll \frac{N^{2 \gamma-1}}{(\log N)^{2}} \quad \text { при } \quad i=1,2,3,4 .
$$

3.5. Оценка сумм $\boldsymbol{\Omega}_{\mathbf{1}}$ и $\boldsymbol{\Omega}_{\mathbf{2}}$. Начнем с изучения $\Omega_{2}$. Из (64) получаем

$$
f_{l l}^{\prime \prime}(m, l)=\pi_{1}-\pi_{2},
$$

где

$$
\pi_{1}=m^{2} r c(c-1)(m l)^{c-2}, \quad \pi_{2}=m^{2} v(c-1) T(m l)^{c-2}\left(T-(m l)^{c}\right)^{\gamma-2} .
$$

Из (4), (55) и условий

$$
P<m l \leqslant 2 P, \quad N \leqslant T \leqslant N+2
$$

имеем

$$
\left|\pi_{1}\right| \asymp|r| m^{2} N^{1-2 \gamma}, \quad \pi_{2} \asymp v m^{2} N^{-\gamma} .
$$

Из (74) и (77) следует, что существует такая достаточно малая константа $\alpha_{0}>0$, что если $|r| \leqslant \alpha_{0} v N^{\gamma-1}$, то $\left|f_{l l}^{\prime \prime}\right| \asymp v m^{2} N^{-\gamma}$.

Аналогично, из (74) и (77) заключаем, что существует такая достаточно большая константа $A_{0}>0$, что если $|r| \geqslant A_{0} v N^{\gamma-1}$, то $\left|f_{l l}^{\prime \prime}\right| \asymp|r| m^{2} N^{1-2 \gamma}$.

Разобьем сумму $\Omega_{2}$ на четыре суммы в соответствии со значениями $r$ следующим образом:

$$
\Omega_{2}=\Omega_{2,1}+\Omega_{2,2}+\Omega_{2,3}+\Omega_{2,4},
$$

где

$$
\begin{array}{ccc}
\text { в } & \Omega_{2,1}: & |r| \leqslant \alpha_{0} v N^{\gamma-1}, \\
\text { в } & \Omega_{2,2}: & -A_{0} v N^{\gamma-1}<r<-\alpha_{0} v N^{\gamma-1}, \\
\text { в } & \Omega_{2,3}: & \alpha_{0} v N^{\gamma-1}<r<A_{0} v N^{\gamma-1}, \\
\text { в } & \Omega_{2,4}: & A_{0} v N^{\gamma-1} \leqslant|r| \leqslant R .
\end{array}
$$

Заметим, что из (34), (55) и (61) следует, что

$$
v N^{\gamma-1} \ll(\log N)^{3} \ll \frac{R}{\log N} .
$$

Сначала рассмотрим $\Omega_{2,4}$. Имеем

$$
\Omega_{2,4}=\sum_{d \leqslant D} \sum_{h \leqslant H} \frac{1}{h} \sum_{A_{0} v N^{\gamma-1} \leqslant|r| \leqslant R} \sup _{T \in[N, N+2]}\left|U_{2}\right| .
$$

Оценим сумму $U_{2}$, определенную в (67), при условии, что выполняется соотношение (82). Напомним, что константа $A_{0}$ выбирается таким образом, что при $|r| \geqslant A_{0} v N^{\gamma-1}$ равномерно по $l \in(P / m, 2 P / m]$ справедливо соотношение $\left|f_{l l}^{\prime \prime}(m, l)\right| \asymp|r| m^{2} N^{1-2 \gamma}$. Следовательно, мы можем снова воспользоваться теоремой Ван дер Корпута (см. [13; гл. 1, теорема 5]) и учитывая (4), получаем

$$
\sum_{P / m<l \leqslant 2 P / m} e(f(m, l)) \ll \frac{P}{m}\left(|r| m^{2} N^{1-2 \gamma}\right)^{1 / 2}+\left(|r| m^{2} N^{1-2 \gamma}\right)^{-1 / 2} \ll|r|^{1 / 2} N^{1 / 2} .
$$


Тогда из (67) и (70) находим

$$
U_{2} \ll|r|^{1 / 2} N^{1 / 2+\gamma / 3}(\log N) .
$$

Подставим это выражение для $U_{2}$ в (84) и воспользуемся (34), (55) и (61), чтобы получить оценку

$$
\Omega_{2,4} \ll D^{5 / 2} N^{2-7 \gamma / 6+\varepsilon} .
$$

Следовательно из (7) и (24) получаем

$$
\Omega_{2,4} \ll \frac{N^{2 \gamma-1}}{(\log N)^{2}} .
$$

Продолжим с $\Omega_{2,3}$. Мы должны изучить сумму $U_{2}$, определенную в $(67)$, при выполнении условия (81). Чтобы это сделать, воспользуемся (64) и вычислим

$$
\begin{aligned}
f_{l l l}^{\prime \prime \prime}(m, l)= & m^{3} r c(c-1)(c-2)(m l)^{c-3} \\
& +m^{3} v(c-1) T\left(T-(m l)^{c}\right)^{\gamma-3}(m l)^{c-3}\left((2-c) T-(c+1)(m l)^{c}\right) .
\end{aligned}
$$

Находим из (74), (75) и (87)

$$
(c-2) f_{l l}^{\prime \prime}(m, l)-l f_{l l l}^{\prime \prime \prime}(m, l)=v(c-1)(2 c-1) T m^{2 c} l^{2 c-2}\left(T-(m l)^{c}\right)^{\gamma-3} .
$$

Последнее вместе с (4) и (76) влечет

$$
\left|(c-2) f_{l l}^{\prime \prime}(m, l)-l f_{l l l}^{\prime \prime \prime}(m, l)\right| \asymp v m^{2} N^{-\gamma} .
$$

Следовательно, существует такая $\kappa_{0}>0$, зависящая только от константы $c$, что для каждого $l \in(P / m, 2 P / m]$ выполняется по крайней мере одно из следующих неравенств:

$$
\left|f_{l l}^{\prime \prime}(m, l)\right| \geqslant \kappa_{0} v m^{2} N^{-\gamma}
$$

или

$$
\left|f_{l l l}^{\prime \prime \prime}(m, l)\right| \geqslant \kappa_{0} v m^{3} N^{-2 \gamma} .
$$

Мы намереваемся показать, что интервал $(P / m, 2 P / m]$ может быть разбит не более чем на семь интервалов таких, что если $J$ есть один из них, то выполняется по крайней мере одно из следующих утверждений:

$$
\begin{aligned}
& \text { имеем (88) для всех } l \in J, \\
& \text { имеем (89) для всех } l \in J .
\end{aligned}
$$

Для установления этого достаточно показать, что уравнение $\left|f_{l l}^{\prime \prime}(m, l)\right|=\kappa_{0} v m^{2} N^{-\gamma}$ имеет не более шести решений в вещественных числах $l \in(P / m, 2 P / m)$. Следовательно, достаточно показать, что если $C$ не зависит от $l$, то уравнение $f_{l l}^{\prime \prime}(m, l)=C$ имеет не более трех решений в вещественных числах $l \in(P / m, 2 P / m)$. Согласно теореме Ролля между любыми двумя решениями последнего уравнения есть решение (в вещественных числах $l$ ) уравнения $f_{l l l}^{\prime \prime \prime}(m, l)=0$. Таки образом, пользуясь $(87)$, мы заключаем, что достаточно показать, что

$$
v T\left(T-(m l)^{c}\right)^{\gamma-3}\left((2-c) T-(c+1)(m l)^{c}\right)=r c(2-c)
$$

имеет не более двух решений для $l \in(P / m, 2 P / m)$, что эквивалентно утверждению, что уравнение

$$
(T-X)^{\gamma-3}((2-c) T-(c+1) X)=\frac{r c(2-c)}{v T}
$$


имеет не более двух решений для $X \in\left(P^{c},(2 P)^{c}\right)$. В качестве альтернативы вместо последнего уравнения можно взглянуть на

$$
(\gamma-3) \log (T-X)+\log ((2-c) T-(c+1) X)=\log \frac{r c(2-c)}{v T} .
$$

Обозначим через $H(X)$ функцию в левой части (92). Из теоремы Ролля мы знаем, что между любыми двумя решениями (92) найдется решение $H^{\prime}(X)=0$. Поскольку

$$
H^{\prime}(X)=\frac{3-\gamma}{T-X}-\frac{c+1}{(2-c) T-(c+1) X},
$$

легко видеть, что $H^{\prime}(X)$ обращается в нуль не более чем при одном значении $X$. Следовательно, (92) имеет не более двух решений в $X$, и наше утверждение доказано.

С другой стороны, из (74), (77) и (87) видим, что при условии на $r$ из (81) имеем

$$
f_{l l}^{\prime \prime}(m, l) \ll v m^{2} N^{-\gamma}, \quad f_{l l l}^{\prime \prime \prime}(m, l) \ll v m^{3} N^{-2 \gamma} .
$$

Отсюда, интервал $(P / m, 2 P / m]$ может быть разбит не более чем на семь таких интервалов , что если $J$ один из них, то выполняется по меньшей мере одно из следующих утверждений:

$$
\begin{aligned}
\left|f_{l l}^{\prime \prime}(m, l)\right| \asymp v m^{2} N^{-\gamma} & & \text { равномерно по } & l \in J, \\
\left|f_{l l l}^{\prime \prime \prime}(m, l)\right| \asymp v m^{3} N^{-2 \gamma} & & \text { равномерно по } & l \in J .
\end{aligned}
$$

Если выполняется (93), то, воспользовавшись теоремой Ван дер Корпута (см. [13; гл. 1, теорема 5]) для второй производной, находим

$$
\sum_{l \in J} e(f(m, l)) \ll \frac{P}{m}\left(v m^{2} N^{-\gamma}\right)^{1 / 2}+\left(v m^{2} N^{-\gamma}\right)^{-1 / 2} \ll v^{1 / 2} N^{\gamma / 2}+v^{-1 / 2} m^{-1} N^{\gamma / 2} .
$$

В случае (94) применим теорему Ван дер Корпута для третьей производной, чтобы получить

$$
\begin{aligned}
\sum_{l \in J} e(f(m, l)) & \ll \frac{P}{m}\left(v m^{3} N^{-2 \gamma}\right)^{1 / 6}+\left(\frac{P}{m}\right)^{1 / 2}\left(v m^{3} N^{-2 \gamma}\right)^{-1 / 6} \\
& \ll v^{1 / 6} m^{-1 / 2} N^{2 \gamma / 3}+v^{-1 / 6} m^{-1} N^{5 \gamma / 6}
\end{aligned}
$$

Отсюда, в любом случае $\sum_{l \in J} e(f(m, l))$ можно оценить суммой выражений из правых частей вышеприведенных неравенств. Следовательно,

$$
\sum_{P / m<l \leqslant 2 P / m} e(f(m, l)) \ll v^{1 / 2} N^{\gamma / 2}+v^{-1 / 2} m^{-1} N^{\gamma / 2}+v^{1 / 6} m^{-1 / 2} N^{2 \gamma / 3}+v^{-1 / 6} m^{-1} N^{5 \gamma / 6} .
$$

Тогда из (4), (67) и (70) находим, что

$$
U_{2} \ll(\log N)^{2}\left(v^{1 / 2} N^{5 \gamma / 6}+v^{-1 / 2} N^{\gamma / 2}+v^{1 / 6} N^{5 \gamma / 6}+v^{-1 / 6} N^{5 \gamma / 6}\right) .
$$

Воспользовавшись (34), (55), (81), (83) и (97), получаем

$$
\begin{aligned}
\Omega_{2,3} & =\sum_{d \leqslant D} \sum_{h \leqslant H} \frac{1}{h} \sum_{\alpha_{0} v N^{\gamma-1}<r<A_{0} v N^{\gamma-1}} \sup _{T \in[N, N+2]}\left|U_{2}\right| \\
& \ll N^{\varepsilon} \sum_{d \leqslant D} \sum_{h \leqslant H} \frac{1}{h}\left(\frac{h^{1 / 2} N^{5 \gamma / 6}}{d^{1 / 2}}+\frac{h^{-1 / 2} N^{\gamma / 2}}{d^{-1 / 2}}+\frac{h^{1 / 6} N^{5 \gamma / 6}}{d^{1 / 6}}+\frac{h^{-1 / 6} N^{5 \gamma / 6}}{d^{-1 / 6}}\right) \\
& \ll N^{\varepsilon}\left(D N^{1 / 2+\gamma / 3}+D^{3 / 2} N^{\gamma / 2}+D N^{1 / 6+2 \gamma / 3}+D^{7 / 6} N^{5 \gamma / 6}\right),
\end{aligned}
$$


и из (7) и (24) мы заключаем, что

$$
\Omega_{2,3} \ll \frac{N^{2 \gamma-1}}{(\log N)^{2}}
$$

Рассмотрим $\Omega_{2,1}$. Мы выбрали постоянную $\alpha_{0}$ таким образом, что из (76) и из условия на $r$ из (79) следует, что

$$
\left|f_{l l}^{\prime \prime}(m, l)\right| \asymp v m^{2} N^{-\gamma} \quad \text { равномерно по } \quad l \in\left(\frac{P}{m}, \frac{2 P}{m}\right] .
$$

Следовательно, сумму $\sum_{P / m<l \leqslant 2 P / m} e(f(m, l))$ можно оценить выражением из правой части (95), и, конечно, оценка (96) выполняется снова. Отсюда видим, что $\Omega_{2,1}$ можно оценить таким же образом, как $\Omega_{2,3}$, т.е.

$$
\Omega_{2,1} \ll \frac{N^{2 \gamma-1}}{(\log N)^{2}} .
$$

Сумма $\Omega_{2,2}$ может быть исследована тем же путем. Из (74)-(76) и (80) следует, что $\left|f_{l l}^{\prime \prime}(m, l)\right| \asymp v m^{2} N^{-\gamma}$, и оценка (96) снова верна. Поэтому

$$
\Omega_{2,2} \ll \frac{N^{2 \gamma-1}}{(\log N)^{2}} .
$$

Из (78), (86) и (99)-(101) заключаем, что

$$
\Omega_{2} \ll \frac{N^{2 \gamma-1}}{(\log N)^{2}} .
$$

Теперь рассмотрим $\Omega_{1}$. Для $U_{1}$ из (66) мы применим преобразование Абеля, чтобы избавиться от множителя $\log l$ во внутренней сумме. Затем мы работаем, как при оценивании $\Omega_{2}$, чтобы получить

$$
\Omega_{1} \ll \frac{N^{2 \gamma-1}}{(\log N)^{2}} .
$$

3.6. Оценка сумм $\boldsymbol{\Omega}_{\mathbf{3}}$ и $\boldsymbol{\Omega}_{\mathbf{4}}$ и завершение доказательства. Рассмотрим сумму $\Omega_{4}$ из (72). Разобьем сумму $U_{4}$ из $(69)$ на $O(\log N)$ сумм вида

$$
W_{M, L}=\sum_{L<l \leqslant 2 L} b(l) \sum_{\substack{M<m \leqslant 2 M \\ P / l<m \leqslant 2 P / l}} a(m) e(f(m, l)),
$$

где

$$
a(m) \ll N^{\varepsilon}, \quad b(l) \ll N^{\varepsilon}, \quad P^{1 / 3} \ll M \ll P^{1 / 2} \ll L \ll P^{2 / 3}, \quad M L \asymp P .
$$

(Если $M \gg P^{1 / 2}$, то меняем местами $m$ и $l$ и соответственно $M$ и $L$ и снова получаем сумму, параметры которой удовлетворяют условия (105).)

Из (104), (105) и неравенства Коши находим

$$
\left|W_{M, L}\right|^{2} \ll N^{\varepsilon} L \sum_{L<l \leqslant 2 L}\left|\sum_{M_{1}<m \leqslant M_{2}} a(m) e(f(m, l))\right|^{2},
$$

где

$$
M_{1}=\max \left(M, \frac{P}{l}\right), \quad M_{2}=\min \left(2 M, \frac{2 P}{l}\right) .
$$


Применим теперь известное неравенство

$$
\left|\sum_{a<m \leqslant b} \xi(m)\right|^{2} \leqslant \frac{b-a+Q}{Q} \sum_{|q|<Q}\left(1-\frac{|q|}{Q}\right) \sum_{\substack{m \in(a, b] \\ m \in(a-q, b-q]}} \xi(m+q) \overline{\xi(m)},
$$

где $Q \in \mathbb{N}, a, b \in \mathbb{R}, 1 \leqslant b-a$ и $\xi(m)$ произвольная комплексная функция. (Доказательство может быть найдено в [15; лемма 8.17].) У нас $\xi(m)=a(m) e(f(m, l)), a=M_{1}, b=M_{2}$. Точное значение $Q$ будет выбрано позднее. Теперь же нам требуется только, чтобы

$$
Q \leqslant M
$$

Тогда находим

$$
\left|W_{M, L}\right|^{2} \ll N^{\varepsilon} L \sum_{L<l \leqslant 2 L} \frac{M}{Q} \sum_{|q| \leqslant Q}\left(1-\frac{|q|}{Q}\right) \sum_{\substack{M_{1}<m \leqslant M_{2} \\ M_{1}<m+q \leqslant M_{2}}} a(m+q) \overline{a(m)} e(f(m+q, l)-f(m, l)) .
$$

Оценим вклад от членов с $q=0$, а затем изменим порядок суммирования, и пользуясь (105) и (107), найдем

$$
\left|W_{M, L}\right|^{2} \ll \frac{N^{\varepsilon}(L M)^{2}}{Q}+\frac{N^{\varepsilon} L M}{Q} \sum_{0<|q| \leqslant Q} \sum_{\substack{M<m \leqslant 2 M \\ M<m+q \leqslant 2 M}}\left|\sum_{L_{1}<l \leqslant L_{2}} e\left(Y_{m, q}(l)\right)\right|,
$$

где

$$
L_{1}=\max \left(L, \frac{P}{m}, \frac{P}{m+q}\right), \quad L_{2}=\min \left(2 L, \frac{2 P}{m}, \frac{2 P}{m+q}\right)
$$

и

$$
Y(l)=Y_{m, q}(l)=f(m+q, l)-f(m, l) .
$$

Теперь легко видеть, что сумма по отрицательным $q$ в формуле (110) равна сумме по положительным $q$, поэтому получаем

$$
\left|W_{M, L}\right|^{2} \ll \frac{N^{\varepsilon}(L M)^{2}}{Q}+\frac{N^{\varepsilon} L M}{Q} \sum_{1 \leqslant q \leqslant Q} \sum_{M<m \leqslant 2 M-q} \mid \sum_{L_{1}<l \leqslant L_{2}} e\left(Y_{m, q}(l)\right) .
$$

Рассмотрим функцию $Y(l)$. Пользуясь (63), (64) и (112), находим, что

$$
Y(l)=\int_{m}^{m+q} f_{t}^{\prime}(t, l) d t=\int_{m}^{m+q} l \phi^{\prime}(t l) d t
$$

и, следовательно,

$$
Y^{\prime \prime}(l)=\int_{m}^{m+q}\left(2 t \phi^{\prime \prime}(t l)+l t^{2} \phi^{\prime \prime \prime}(t l)\right) d t, \quad Y^{\prime \prime \prime}(l)=\int_{m}^{m+q}\left(3 t^{2} \phi^{\prime \prime \prime}(t l)+l t^{3} \phi^{(4)}(t l)\right) d t .
$$

Из (63) и (115) получаем

$$
Y^{\prime \prime}(l)=\int_{m}^{m+q}\left(\Phi_{1}(t)-\Phi_{2}(t)\right) d t
$$

где

$$
\begin{aligned}
& \Phi_{1}(t)=r c^{2}(c-1) t^{c-1} l^{c-2}, \\
& \Phi_{2}(t)=v(c-1) T t^{c-1} l^{c-2}\left(T-(t l)^{c}\right)^{\gamma-3}\left(c T+(c-1)(t l)^{c}\right) .
\end{aligned}
$$


Напомним, что $P$ задано в (4), $r$ есть переменная суммирования в $(72)$ и $v$ определяется из (55). Если $t \in[m, m+q]$, то $t l \asymp P$. Таким образом, из условия

$$
N \leqslant T \leqslant N+2
$$

и определения $P$ находим, что равномерно по $t \in[m, m+q]$ справедливы соотношения

$$
\left|\Phi_{1}(t)\right| \asymp|r| m N^{1-2 \gamma}, \quad \Phi_{2}(t) \asymp v m N^{-\gamma} .
$$

Из (116) и (120) видим, что существует такая достаточно малая константа $\alpha_{1}>0$, что если $|r| \leqslant \alpha_{1} v N^{\gamma-1}$, то $\left|Y^{\prime \prime}(l)\right| \asymp q v m N^{-\gamma}$. Аналогично, мы заключаем, что существует такая достаточно большая константа $A_{1}>0$, что если $|r| \geqslant A_{1} v N^{\gamma-1}$, то $\left|Y^{\prime \prime}(l)\right| \asymp|r| q m N^{1-2 \gamma}$. Поэтому имеет смысл разбить сумму $\Omega_{4}$ на четыре суммы согласно значению $r$ следующим образом:

$$
\Omega_{4}=\Omega_{4,1}+\Omega_{4,2}+\Omega_{4,3}+\Omega_{4,4},
$$

где

$$
\begin{array}{ccc}
\text { в } & \Omega_{4,1}: & |r| \leqslant \alpha_{1} v N^{\gamma-1}, \\
\text { в } & \Omega_{4,2}: & -A_{1} v N^{\gamma-1}<r<-\alpha_{1} v N^{\gamma-1}, \\
\text { в } & \Omega_{4,3}: & \alpha_{1} v N^{\gamma-1}<r<A_{1} v N^{\gamma-1}, \\
\text { в } & \Omega_{4,4}: & A_{1} v N^{\gamma-1} \leqslant|r| \leqslant R .
\end{array}
$$

Сначала рассмотрим $\Omega_{4,4}$. Из $(72)$ и (125) имеем

$$
\Omega_{4,4} \ll(\log N) \sum_{d \leqslant D} \sum_{h \leqslant H} \frac{1}{h} \sum_{A_{1} v N^{\gamma-1} \leqslant|r| \leqslant R} \sup _{\substack{T \in[N, N+2] \\ M, L:(105)}}\left|W_{M, L}\right| .
$$

(Супремум берется по $T \in[N, N+2]$ и $M, L$, удовлетворяющим условиям из (105).)

Рассмотрим сумму $W_{M, L}$. Уже упоминалось, что если $|r| \geqslant A_{1} v N^{\gamma-1}$, то равномерно по $l \in$ $\left(L_{1}, L_{2}\right]$ имеем $Y^{\prime \prime}(l) \asymp|r| q m N^{1-2 \gamma}$. Поэтому можно использовать теорему Ван дер Корпута (см. [13; гл. 1 , теорема 5]) для второй производной и из (4), (105) и (111) получить

$$
\sum_{L_{1}<l \leqslant L_{2}} e(Y(l)) \ll L\left(|r| q m N^{1-2 \gamma}\right)^{1 / 2}+\left(|r| q m N^{1-2 \gamma}\right)^{-1 / 2} \ll|r|^{1 / 2} q^{1 / 2} M^{-1 / 2} N^{1 / 2} .
$$

Тем самым, из (4), (105) и (113) находим

$$
W_{M, L} \ll N^{\varepsilon}\left(N^{\gamma} Q^{-1 / 2}+|r|^{1 / 4} Q^{1 / 4} N^{1 / 4+5 \gamma / 8}\right) .
$$

Из (34), (61) и (126) имеем

$$
\begin{aligned}
\Omega_{4,4} & \ll N^{\varepsilon} \sum_{d \leqslant D} \sum_{h \leqslant H} \frac{1}{h} \sum_{|r| \leqslant d N^{1-\gamma}(\log N)^{12}}\left(N^{\gamma} Q^{-1 / 2}+|r|^{1 / 4} Q^{1 / 4} N^{1 / 4+5 \gamma / 8}\right) \\
& \ll N^{\varepsilon}\left(D^{2} N Q^{-1 / 2}+Q^{1 / 4} D^{9 / 4} N^{3 / 2-5 \gamma / 8}\right) .
\end{aligned}
$$

Мы выберем

$$
Q=\left[D^{-1 / 3} N^{5 \gamma / 6-2 / 3}\right]
$$

Теперь легко проверить, что условие (109) выполняется. Следовательно, из (7) и (24) получаем

$$
\Omega_{4,4} \ll \frac{N^{2 \gamma-1}}{(\log N)^{2}} .
$$


Теперь рассмотрим $\Omega_{4,3}$. Из $(72)$ и (124) имеем

$$
\Omega_{4,3} \ll(\log N) \sum_{d \leqslant D} \sum_{h \leqslant H} \frac{1}{h} \sum_{\alpha_{1} v N^{\gamma-1}<r<A_{1} v N^{\gamma-1}} \sup _{\substack{T \in[N, N+2] \\ M, L:(105)}}\left|W_{M, L}\right| .
$$

Рассмотрим сумму $W_{M, L}$ из вышеприведенного выражения. Пользуясь (116)-(118), находим

$$
Y^{\prime \prime \prime}(l)=\int_{m}^{m+q}\left(\Psi_{1}(t)+\Psi_{2}(t)\right) d t,
$$

где

$$
\begin{aligned}
& \Psi_{1}(t)=r c^{2}(c-1)(c-2) t^{c-1} l^{c-3} \\
& \Psi_{2}(t)=v(c-1) T t^{c-1} l^{c-3}\left(T-(t l)^{c}\right)^{\gamma-4}\left(c(2-c) T^{2}+\left(-4 c^{2}+3 c-2\right) T(t l)^{c}+\left(1-c^{2}\right)(t l)^{2 c}\right) .
\end{aligned}
$$

Из (116)-(118) и (131)-(133) получаем

$$
l Y^{\prime \prime \prime}(l)+(2-c) Y^{\prime \prime}(l)=-\int_{m}^{m+q} \Theta(t) d t,
$$

где

$$
\Theta(t)=v(c-1) T t^{2 c-1} l^{2 c-2}\left(T-(t l)^{c}\right)^{\gamma-4}\left(2 c(2 c-1) T+\left(2 c^{2}-3 c+1\right)(t l)^{c}\right) .
$$

Пользуясь $(4),(105)$ и $(119)$, находим, что равномерно по $t \in[m, m+q]$ справедливо соотношение

$$
\Theta(t) \asymp v m N^{-\gamma}
$$

Отсюда

$$
\left|(2-c) Y^{\prime \prime}(l)+l Y^{\prime \prime \prime}(l)\right| \asymp q m v N^{-\gamma}
$$

равномерно по $l \in\left(L_{1}, L_{2}\right]$. Следовательно, существует $\kappa_{1}>0$, которая зависит только от $c$ и такая, что по крайней мере одно из следующих неравенств выполняется для любого $l \in\left(L_{1}, L_{2}\right]:$

$$
\begin{aligned}
\left|Y^{\prime \prime}(l)\right| & \geqslant \kappa_{1} v q m N^{-\gamma}, \\
\left|Y^{\prime \prime \prime}(l)\right| & \geqslant \kappa_{1} v q m^{2} N^{-2 \gamma} .
\end{aligned}
$$

На следующем шаге будет показано, что интервал $\left(L_{1}, L_{2}\right]$ может быть разбит не более чем на 13 интервалов таких, что если $J$ - один из них, то по крайней мере одно из следующих утверждений выполняется:

$$
\begin{aligned}
& \text { имеет место (134) для всех } l \in J, \\
& \text { имеет место (135) для всех } l \in J .
\end{aligned}
$$

Чтобы это установить, достаточно показать, что уравнение $\left|Y^{\prime \prime}(l)\right|=\kappa_{1} v q m N^{-\gamma}$ имеет не более 12 решений в вещественных числах $l \in\left(L_{1}, L_{2}\right)$. То есть, достаточно показать, что если $C$ не зависит от $l$, то уравнение $Y^{\prime \prime}(l)=C$ имеет не более шести решений в вещественных числах $l \in\left(L_{1}, L_{2}\right)$. Согласно теореме Ролля между любыми двумя решениями последнего уравнения найдется решение уравнения

$$
Y^{\prime \prime \prime}(l)=0
$$

Поэтому достаточно показать, что (138) имеет не более пяти решений в вещественных числах $l \in\left(L_{1}, L_{2}\right)$. 
Легко видеть из (87) и (112), что (138) эквивалентно уравнению

$$
\begin{gathered}
(m+q)^{c}\left(T-(m+q)^{c} l^{c}\right)^{\gamma-3}\left((2-c) T-(c+1)(m+q)^{c} l^{c}\right) \\
-m^{c}\left(T-m^{c} l^{c}\right)^{\gamma-3}\left((2-c) T-(c+1) m^{c} l^{c}\right) \\
=\frac{r c(2-c)\left((m+q)^{c}-m^{c}\right)}{v T} .
\end{gathered}
$$

Пусть $X=l^{c}$. Определим

$$
\begin{gathered}
\mathcal{F}(X)=(m+q)^{c}\left(T-(m+q)^{c} X\right)^{\gamma-3}\left((2-c) T-(c+1)(m+q)^{c} X\right) \\
-m^{c}\left(T-m^{c} X\right)^{\gamma-3}\left((2-c) T-(c+1) m^{c} X\right) .
\end{gathered}
$$

Будет достаточно показать, что если $B$ не зависит от $X$, то уравнение $\mathcal{F}(X)=B$ имеет не более пяти решений с $X \in\left(L_{1}^{c}, L_{2}^{c}\right)$.

Еще раз обращаемся к теореме Ролля, чтобы подтвердить, что достаточно доказать, что уравнение $\mathcal{F}^{\prime}(X)=0$ имеет не более четырех решений при $X \in\left(L_{1}^{c}, L_{2}^{c}\right)$. Можно записать $\mathcal{F}^{\prime}(X)=0$ как

$$
\begin{gathered}
(m+q)^{2 c}\left(T-(m+q)^{c} X\right)^{\gamma-4}\left((4 c+2 \gamma-6) T+(2 c-\gamma+1)(m+q)^{c} X\right) \\
=m^{2 c}\left(T-m^{c} X\right)^{\gamma-4}\left((4 c+2 \gamma-6) T+(2 c-\gamma+1) m^{c} X\right),
\end{gathered}
$$

что, в свою очередь, эквивалентно

$$
\mathcal{G}(X)=\log m^{c}-\log (m+q)^{c},
$$

где функция $\mathcal{G}(X)$ определяется из

$$
\begin{gathered}
\mathcal{G}(X)=(\gamma-4) \log \left(T-(m+q)^{c} X\right)+\log \left((4 c+2 \gamma-6) T+(2 c+1-\gamma)(m+q)^{c} X\right) \\
-(\gamma-4) \log \left(T-m^{c} X\right)-\log \left((4 c+2 \gamma-6) T+(2 c+1-\gamma) m^{c} X\right) .
\end{gathered}
$$

По той же причине, что и до этого, достаточно установить, что уравнение

$$
\mathcal{G}^{\prime}(X)=0
$$

имеет не более трех решений при $X \in\left(L_{1}^{c}, L_{2}^{c}\right)$. Это легко показать, поскольку

$$
\begin{gathered}
\mathcal{G}^{\prime}(X)=\frac{(4-\gamma)(m+q)^{c}}{T-(m+q)^{c} X}+\frac{(2 c+1-\gamma)(m+q)^{c}}{(4 c+2 \gamma-6) T+(2 c+1-\gamma)(m+q)^{c} X} \\
-\frac{(4-\gamma) m^{c}}{T-m^{c} X}-\frac{(2 c+1-\gamma) m^{c}}{(4 c+2 \gamma-6) T+(2 c+1-\gamma) m^{c} X} .
\end{gathered}
$$

Следовательно, число решений (139) не превосходит числа корней многочлена степени не выше 3. Этот многочлен отличен от нуля, и можно легко в этом убедиться, проверив, что $\mathcal{G}^{\prime}(0) \neq 0$. Мы оставляем эти простые вычисления читателю.

С другой стороны из (116), (120), (124) и (131)-(133) имеем

$$
Y^{\prime \prime}(l) \ll v q m N^{-\gamma}, \quad Y^{\prime \prime \prime}(l) \ll v q m^{2} N^{-2 \gamma} .
$$

Поэтому мы заключаем, что интервал $\left(L_{1}, L_{2}\right]$ может быть разбит на не более чем 13 таких интервалов, что если $J$ - один из них, то имеет место по крайней мере одно из следующих утверждений:

$$
\begin{aligned}
\left|Y^{\prime \prime}(l)\right| \asymp v q m N^{-\gamma} & & \text { равномерно по } & l \in J, \\
\left|Y^{\prime \prime \prime}(l)\right| \asymp v q m^{2} N^{-2 \gamma} & & \text { равномерно по } & l \in J .
\end{aligned}
$$


Если (141) выполняется, то воспользуемся (4), (105) и теоремой Ван дер Корпута (см. [13; гл. 1, теорема 5]) для второй производной, чтобы получить

$$
\begin{aligned}
\sum_{l \in J} e(Y(l)) & \ll L\left(q v m N^{-\gamma}\right)^{1 / 2}+\left(q v m N^{-\gamma}\right)^{-1 / 2} \\
& \ll q^{1 / 2} v^{1 / 2} L M^{1 / 2} N^{-\gamma / 2}+q^{-1 / 2} v^{-1 / 2} M^{-1 / 2} N^{\gamma / 2} .
\end{aligned}
$$

В случае, когда (142) удовлетворяется, применим (4), (105) и теорему Ван дер Корпута для третьей производной и получим

$$
\begin{aligned}
\sum_{l \in J} e(Y(l)) & \ll L\left(q v m^{2} N^{-2 \gamma}\right)^{1 / 6}+L^{1 / 2}\left(q v m^{2} N^{-2 \gamma}\right)^{-1 / 6} \\
& \ll q^{1 / 6} v^{1 / 6} L M^{1 / 3} N^{-\gamma / 3}+q^{-1 / 6} v^{-1 / 6} L^{1 / 2} M^{-1 / 3} N^{\gamma / 3}
\end{aligned}
$$

Следовательно, в каждом случае $\sum_{l \in J} e(Y(l))$ можно оценить с помощью суммы выражений из правых частей неравенств (143) и (144). Отсюда получаем

$$
\begin{aligned}
& \sum_{L_{1}<l \leqslant L_{2}} e(Y(l)) \ll q^{1 / 2} v^{1 / 2} L M^{1 / 2} N^{-\gamma / 2}+q^{-1 / 2} v^{-1 / 2} M^{-1 / 2} N^{\gamma / 2} \\
&+q^{1 / 6} v^{1 / 6} L M^{1 / 3} N^{-\gamma / 3}+q^{-1 / 6} v^{-1 / 6} L^{1 / 2} M^{-1 / 3} N^{\gamma / 3} .
\end{aligned}
$$

Пользуясь (4), (105), (111) и (113), находим, что

$$
\begin{array}{r}
W_{M, L} \ll N^{\varepsilon}\left(N^{\gamma} Q^{-1 / 2}+v^{1 / 4} Q^{1 / 4} N^{7 \gamma / 8}+v^{-1 / 4} Q^{-1 / 4} N^{7 \gamma / 8}\right. \\
\left.+v^{1 / 12} Q^{1 / 12} N^{11 \gamma / 12}+v^{-1 / 12} Q^{-1 / 12} N^{23 \gamma / 24}\right) .
\end{array}
$$

Применим вышеприведенную оценку для $W_{M, L}$ в (130). Тогда из (34) и (55) получаем

$$
\begin{gathered}
\Omega_{4,3} \ll N^{\varepsilon} \sum_{d \leqslant D} \sum_{h \leqslant H} \frac{1}{h} \sum_{r<A_{1} \log ^{3} N}\left(N^{\gamma} Q^{-1 / 2}+\left(\frac{h}{d}\right)^{1 / 4} Q^{1 / 4} N^{7 \gamma / 8}+\left(\frac{h}{d}\right)^{-1 / 4} Q^{-1 / 4} N^{7 \gamma / 8}\right. \\
\left.+\left(\frac{h}{d}\right)^{1 / 12} Q^{1 / 12} N^{11 \gamma / 12}+\left(\frac{h}{d}\right)^{-1 / 12} Q^{-1 / 12} N^{23 \gamma / 24}\right) \\
\ll N^{\varepsilon}\left(D N^{\gamma} Q^{-1 / 2}+D Q^{1 / 4} N^{1 / 4+5 \gamma / 8}+D^{5 / 4} Q^{-1 / 4} N^{7 \gamma / 8}\right. \\
\left.+D Q^{1 / 12} N^{1 / 12+5 \gamma / 6}+D^{13 / 12} Q^{-1 / 12} N^{23 \gamma / 24}\right) .
\end{gathered}
$$

С тем выбором $Q$, который мы сделали в (128), теперь ясно, что

$$
\Omega_{4,3} \ll \frac{N^{2 \gamma-1}}{(\log N)^{2}} .
$$

Теперь продолжим с изучением $\Omega_{4,1}$. Мы выбрали константу $\alpha_{1}$ таким образом, что из $(76)$ и (122) следует, что

$$
Y^{\prime \prime}(l) \asymp v q m N^{-\gamma} \quad \text { равномерно по } \quad l \in\left(L_{1}, L_{2}\right] .
$$

Тогда сумма $\sum_{P / m<l \leqslant 2 P / m} e(Y(l))$ может оцениваться выражением из правой части (143). Это наблюдение показывает, что $\Omega_{4,1}$ оценивается той же величиной, что и $\Omega_{4,3}$, т.е.

$$
\Omega_{4,1} \ll \frac{N^{2 \gamma-1}}{(\log N)^{2}} .
$$


Очень похожим образом можно показать, что

$$
\Omega_{4,2} \ll \frac{N^{2 \gamma-1}}{(\log N)^{2}} .
$$

Тогда из (121), (129) и (145)-(147) получаем

$$
\Omega_{4} \ll \frac{N^{2 \gamma-1}}{(\log N)^{2}} .
$$

Остается найти оценку для $\Omega_{3}$. Тот же аргумент, что и для $\Omega_{4}$, может быть применен здесь еще раз, чтобы показать, что

$$
\Omega_{3} \ll \frac{N^{2 \gamma-1}}{(\log N)^{2}} .
$$

Из (102), (103), (148) и (149) заключаем, что (73) выполнено, и теорема доказана.

\section{Список литературы}

[1] И. И. Пятецкий-Шапиро, "О распределении простых чисел в последовательностях вида $[f(n)]$ ", Матем. сб., 33 (75):3 (1953), 559-566.

[2] J. Rivat, J. Wu, "Prime numbers of the form [nc]", Glasgow Math. J., 43:2 (2001), 237-254.

[3] X. Wang, Y. Cai, "An additive problem involving Piatetski-Shapiro primes", Int. J. Number Theory, 7:5 (2011), 1359-1378.

[4] T. P. Peneva, "An Additive problem with Piatetski-Shapiro primes and almost-primes", Monatsh. Math., 140:2 (2003), 119-133.

[5] R. C. Baker, W.D. Banks, Z. V. Guo, A. M. Yeager, "Piatetski-Shapiro primes from almost primes", Monatsh. Math., 174:3 (2014), 357-370.

[6] J.-M. Deshouillers, "Un problème binaire en théorie additive", Acta Arith., 25 (1974), 393-403.

[7] С. А. Гриценко, “Три аддитивные задачи”, Изв. РАН. Сер. матем., 56:6 (1992), 1198-1216.

[8] С.В. Конягин, “Аддитивная проблема с дробными степенями”, Матем. заметки, 73:4 (2003), 633-636.

[9] A. V. Kumchev, "A binary additive equation involving fractional powers", Int. J. Number Theory, 5:2 (2009), 281-292.

[10] J. R. Chen, "On the representation of a larger even integer as the sum of a prime and the product of at most two primes", Sci. Sinica, 16 (1973), 157-176.

[11] G. Greaves, Sieves in Number Theory, Ergeb. Math. Grenzgeb. (3), 43, Springer-Verlag, Berlin, 2001.

[12] J.D. Vaaler, "Some extremal problems in Fourier analysis", Bull. Amer. Math. Soc. (N.S.), 12:2 (1985), 183-216.

[13] А. А. Карацуба, Основы аналитической теории чисел, Наука, М., 1983.

[14] R. C. Vaughan, "An elementary method in prime number theory", Acta Arith., 37 (1980), 111-115.

[15] H. Iwaniec, E. Kowalski, Analytic Number Theory, Amer. Math. Soc. Colloq. Publ., 53, Amer. Math. Soc., Providence, RI, 2004. 